\title{
A laboratory evaluation of alcohols as attractants for the sandfly Lutzomyia longipalpis (Diptera:Psychodidae)
}

\author{
Jairo Torres Magalhães-Junior ${ }^{1}$, Stella Maria Barrouin-Melo', Arlene Gonçalves Corrêa², \\ Flavia Benini da Rocha Silva ${ }^{3}$, Vicente Estevam Machado ${ }^{3}$, José Silvio Govone ${ }^{4}$ and Mara Cristina Pinto ${ }^{3 *}$
}

\begin{abstract}
Background: The potential attraction from 1-octen-3-ol for sandflies has been documented; however, studies using other primary alcohols are limited.

Findings: We used a wind tunnel to compare the activation and attractive behaviors in male and female Lutzomyia longipalpis using 1-octen-3-ol and three additional alcohols, 1-octanol, 1-heptanol and 1-nonanol at three different concentrations: neat (100\%) and diluted in hexane (10\% and 50\%). The compounds 1-octen-3-ol and 1-nonanol induced a clear concentration-dependent activation and attraction response in females. In males, 1-octen-3-ol, 1-nonanol and 1-heptanol yielded the same results.
\end{abstract}

Conclusions: L. longipalpis is attracted to 1-octen-3-ol, 1-nonanol and 1-heptanol, which are found in many plant volatiles.

Keywords: Sandflies, Phlebotomine, Kairomone, Octenol, Octanol, Heptanol, Nonanol, Attraction, Wind tunnel

\section{Findings}

\section{Background}

Volatile compounds used as haematophagous insect lures may improve the efficacy of traps for surveillance and control of disease vectors. For the sandfly Lutzomyia longipalpis (Lutz \& Neiva), which is the major Leishmania infantum vector in South America, previous investigations into attractive lures have focused on male pheromones [1] and kairomones [2].

The kairomone 1-octen-3-ol (hereafter octenol) is a volatile component of bovine [3] and human breath [4]. Its potential role as an attractant has been documented for different haematophagous insect species, such as mosquitoes [5] and tsetse flies [3]. For the New World sandfly species, octenol has previously been used with light traps and found to be relatively attractive to Psathyromyia shannoni (Dyar) (=Lutzomyia shannoni) [6] and, in a concentration-

\footnotetext{
* Correspondence: marap@fcfar.unesp.br

${ }^{3}$ Departamento de Ciências Biológicas, Faculdade de Ciências Farmacêuticas, Universidade Estadual Julio de Mesquita Filho, UNESP, 14801-902 Araraquara, SP, Brazil

Full list of author information is available at the end of the article
}

dependent manner, to Nyssomyia neivai (Lutz \& Neiva) (=Lutzomyia intermedia) [7]. For L. longipalpis, octenol elicited significant olfactory responses in electrophysiological experiments [8], but it showed a weak attractive response at $0.5 \mathrm{mg} / \mathrm{h}$ associated with light traps under field conditions [2].

Unlike octenol, studies on the potential attractiveness of other primary alcohols, namely, 1-octanol, 1-heptanol, 1nonanol (hereafter, octanol, heptanol and nonanol), for haematophagous insects are limited. These alcohols were identified at small levels in incubated human sweat [9]. Only nonanol has been demonstrated as relatively attractive to Aedes aegypti (Linnaeus) compared with a control [10]. No studies have been reported on attractiveness of these alcohols to sandflies.

The aim of this study was to evaluate L. longipalpis male and female responses to octenol, octanol, heptanol and nonanol at different concentrations using the wind tunnel method. 


\section{Methods}

Insects

Sandflies were collected in Ipecaetá (12¹8'00'S $\left.39^{\circ} 18^{\prime} 28^{\prime \prime} \mathrm{W}\right)$, Bahia State and kept in a colony at the Laboratory of Veterinary Infectious Diseases (Federal University of Bahia) for 18 generations. The insects were maintained in netting cages using standard methods with access to a $50 \%$ sucrose solution at $26 \pm 1^{\circ} \mathrm{C}$ and $80-90 \%$ humidity. The sandfly species names are presented using the Galati classification system [11] followed by the corresponding Young and Duncan nomenclature [12] in brackets when cited for the first time.

\section{Bioassay protocol in the wind tunnel}

Each bioassay was performed from 9:00 to 19:00 in a transparent acrylic wind tunnel (length $200 \mathrm{~cm}$, width $20 \mathrm{~cm}$ and height $20 \mathrm{~cm}$ ) as previously described [13]. For each test, three male or female L. longipalpis were placed inside a releasing chamber for 30 min for acclimation before each test. The insects were 3-6 days old and received only sugar meal. Females had not received a blood meal. The chamber was then placed inside the wind tunnel $50 \mathrm{~cm}$ downwind from the odor source. Each trial was 2 min long, and we recorded the sandfly activation and attraction behaviors. The activation behavior was demonstrated through the number of sandflies that left the releasing chamber. The attraction behavior was demonstrated through the number of sandflies that reached the odor source. Thirty insect specimens were used for each concentration per compound.

The compounds used for the experiments were octenol, octanol, heptanol and nonanol (98.0\%, Aldrich Chemical, Milwaukee, WI) at three different concentrations: neat (100\%) and diluted in hexane (10\% and 50\%). Each concentration was released by placing $200 \mu \mathrm{L}$ onto filter paper $(4 \times 4 \mathrm{~cm})$ in the wind tunnel entrance. The controls were $200 \mu \mathrm{L}$ of hexane on filter paper $(4 \times 4 \mathrm{~cm})$ before each trial.

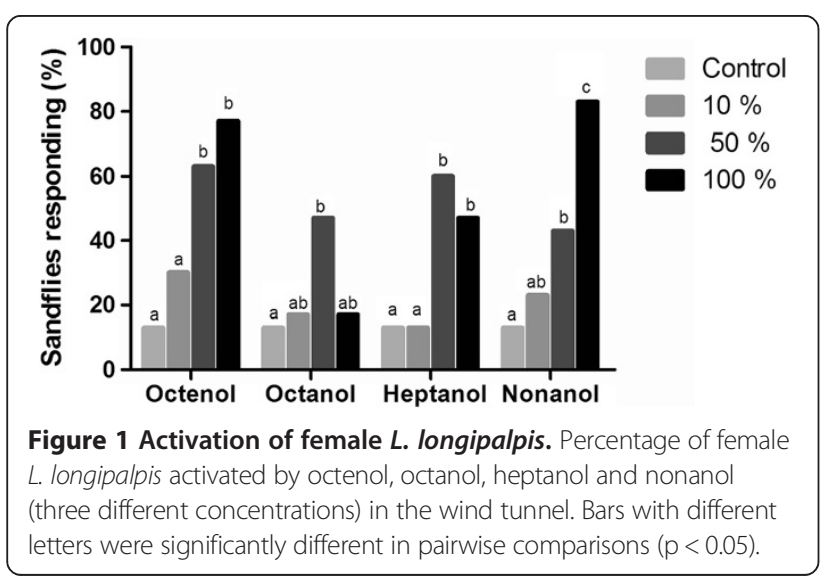

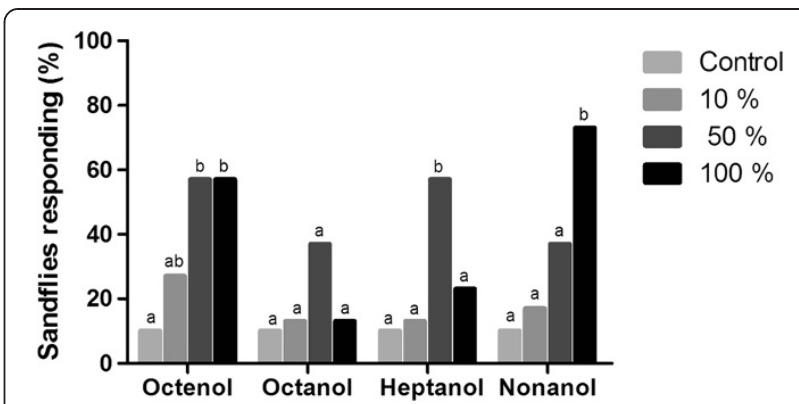

Figure 2 Attraction of female L. longipalpis. Percentage of female L. longipalpis attracted by octenol, octanol, heptanol and nonanol (three different concentrations) in the wind tunnel. Bars with different letters were significantly different in pairwise comparisons $(p<0.05)$

\section{Statistical analysis}

Chi square tests were used to evaluate the different proportions of males and females activated and attracted by each compound. Initially, the test was conducted for all four groups simultaneously. Thereafter, if a significant difference was verified, each of the two groups was compared separately. The statistical analyses were performed using BioEstat (version 5.0; Mamirauá/CNPq, Belém, PA, Brazil).

\section{Results}

\section{Female responses}

Octenol and nonanol induced a clear concentrationdependent activation and attraction response within the dosage range evaluated. For octenol, the activation and attraction responses were significantly different at the $50 \%$ concentration compared with the control $(\mathrm{p}<0.05)$, but the $50 \%$ and $100 \%$ concentrations were not different ( $p>0.05)$. For nonanol, the activation response was statistically different from the $50 \%$ concentration compared with the control; however, there was a significantly different attraction response only at the $100 \%$ concentration $(\mathrm{p}<0.05)$ (Figures 1 and 2).

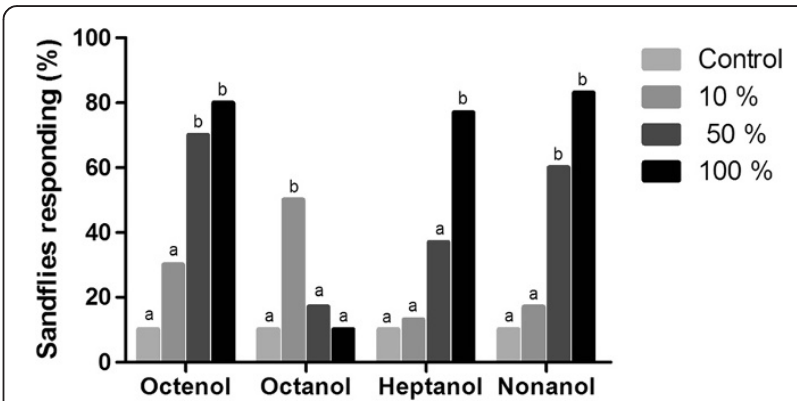

Figure 3 Activation of male L. longipalpis. Percentage of male $L$. longipalpis activated by octenol, octanol, heptanol and nonanol (three different concentrations) in the wind tunnel. Bars with different letters were significantly different in pairwise comparisons $(p<0.05)$ 


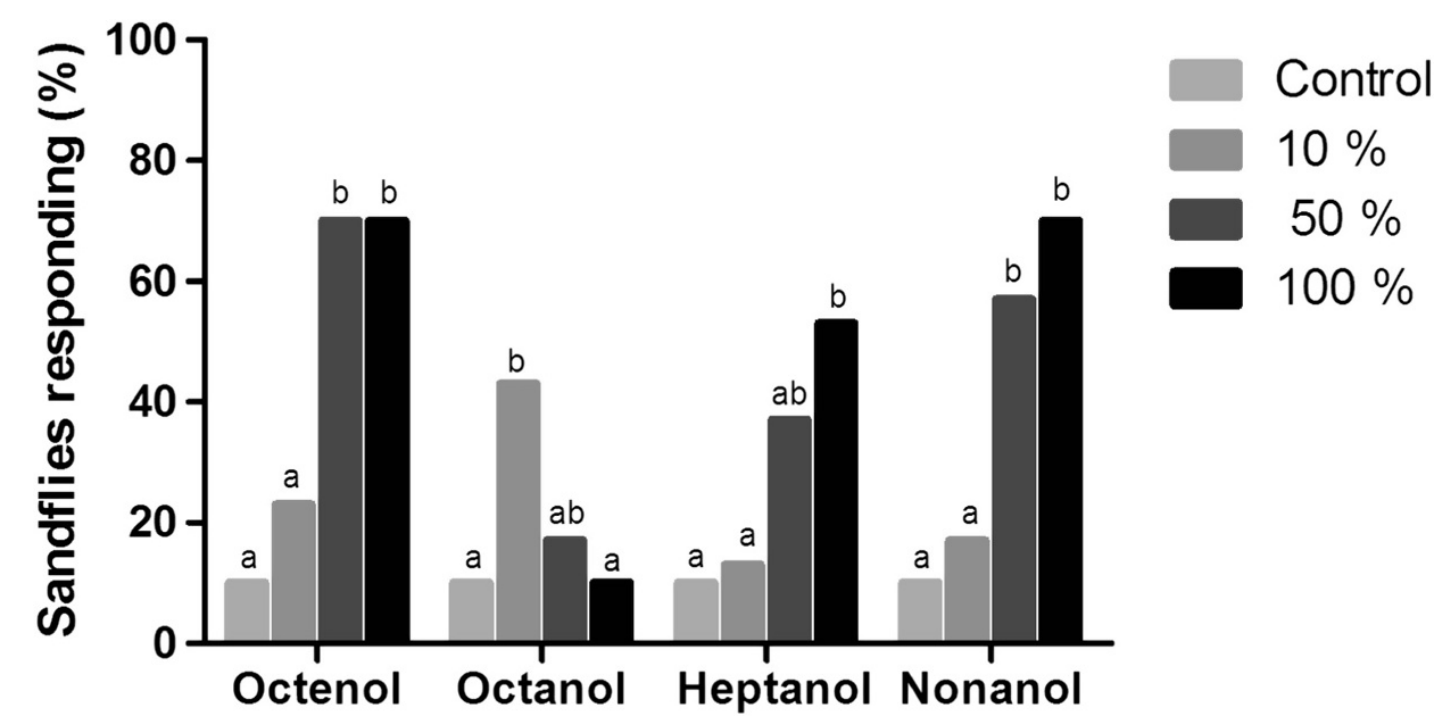

Figure 4 Attraction of male L. longipalpis. Percentage of male L. longipalpis attracted by octenol, octanol, heptanol and nonanol (three different concentrations) in the wind tunnel. Bars with different letters were significantly different in pairwise comparisons $(p<0.05)$.

The female activation and attraction response to octanol did not yield a concentration-dependent pattern. The only statistical difference detected was for activation at the $50 \%$ concentration compared with the control $(\mathrm{p}<0.05)$.

For heptanol, the female activation responses were concentration-dependent; the $100 \%$ response was greater than at $50 \%$ compared with the control $(\mathrm{p}<0.05)$. However, only the $50 \%$ concentration yielded a significantly different insect attraction response compared with the control $(\mathrm{p}<0.05)$; the $100 \%$ concentration was not different from the control $(\mathrm{p}>0.05)$.

\section{Male responses}

The male sandfly responses followed a similar pattern as the females for octenol, nonanol and heptanol (i.e., a concentration-dependent response).

For octanol, similar to the females, the males did not exhibit a concentration-dependent response; however, the only significant difference detected in the activation and attraction response was at the $10 \%$ concentration compared with the control $(\mathrm{p}<0.05)$. For heptanol, although the males presented the same pattern as the females, the best activation and attraction responses were at the highest concentration $(100 \%)$ compared with the control $(\mathrm{p}<0.05)$ (Figures 3 and 4).

\section{Discussion}

Our results show that in addition to octenol, other alcohols evoke sandfly responses and should also be investigated. Octenol and nonanol elicited the highest responses from L. longipalpis females, and octenol, heptanol and nonanol elicited the highest responses from males. Further, not all mosquito species respond equally to octenol [5], and whether different groups of alcohols would increase attractant activity for such species has been discussed [10]. However, a clear structure-activity relationship has not been demonstrated [14].

Octenol's role as a kairomone has been extensively evaluated in haematophagous insects because it is found

Table 1 Reports of haematophagous insect responses to octanol, heptanol and nonanol

\begin{tabular}{lccc}
\hline Compounds & Sources of compounds & Insects & References \\
\hline 1-octanol & Commercial (Aldrich) & Experimental design responses & Sutcliffe et al. [18] \\
1-heptanol & Fetasternal glands of Triatoma brasiliensis & Triatoma brasiliensis & Vitta et al. [19] \\
& Volatiles from cattle headspace and urine & CG- EAD & No response \\
1-nonanol & Haematobia irritans Stomoxys calcitrans & Birkett et al. [20] \\
\hline
\end{tabular}


in different sources, such as bovine [3] and human breath [15]. Octenol's role in attracting sandflies is controversial, but our results showed a clear dosedependent response with weak attractiveness at a low concentration (10\%). These results may explain the poor results from $L$. longipalpis captures in the field when octenol was used at a low concentration (0.5 mg/h) [2].

Nevertheless, primary alcohols, such as octanol, heptanol and nonanol, have not been well-investigated for haematophagous insects, and this is the first report of such a study using sandflies. Such alcohols are not directly associated with vertebrate breath or skin odors, which may be the basis for the lack of interest in their potential role as an attractant for disease vectors. Nonetheless, those alcohols were detected at small levels in human sweat after incubation for 42-52 h, and nonanol presented the highest levels compared with octanol and heptanol [9]. Heptanol was also observed in chicken feather hydrolysate [16], which is relevant for sandflies because they are present at high levels in chicken sheds. Such bird shelters are the putative breeding sites for $L$. longipalpis [17]. The literature reports on haematophagous insect responses to octanol, heptanol and nonanol are summarized in Table 1 [18-20].

Floral volatiles are composed of various substances that have been shown to be attractive to mosquitoes [21]. The primary alcohols herein were identified in several mushrooms species [22] and other herbaceous plants [23]. From an environmental perspective, it is noteworthy that primary alcohols are in plants, which are generally sandfly feed sources. Both male and female sandflies require carbohydrates for energy, which are acquired through feeding directly on plant tissues in the field [24].

Herein, we observed similarities and distinctions between the insect sexes considering their biological responses to the primary alcohols evaluated. Although both males and females require plant sap to survive and specific variation in attraction to their constituent compounds is expected, it is difficult to explain such events through reports on morphological aspects. Differences were also observed in the number of sensilla on the second palpal segment in females (2-6) compared with males (1-2). However, an equal number of sensilla were observed in the third segment of the maxillary palps (Newstead's sensilla) for L. longipalpis males and females [25].

A dose-dependent response to octenol in female L. longipalpis was previously observed through electrophysiological recordings [8]. The female behavioral responses to octenol herein are consistent with such studies. Although no studies have investigated male electrophysiological responses to octenol, they were both activated and attracted in the wind tunnel.
Plant-specific emissions are important for attracting sandflies, and further studies with plant volatiles may be a potential approach to improve sandfly lures.

\section{Competing interests}

The authors declare that they have no competing interests.

\section{Authors' contributions}

JMJ, SM, AC and MP conceived the study and drafted the manuscript. JMJ, MP, FS and VM conducted the bioassay experiments. JG provided statistical analysis. All authors read and approved the final manuscript.

\section{Acknowledgements}

This work was supported by Fundação para o Amparo a Pesquisa do Estado da Bahia-FAPESB (Programa de Apoio a Núcleos Emergentes-Pronem; Apoio à Formação e Articulação de Redes de Pesquisa no Estado da Bahia; and scholarship for JMJ). MP received a grant from the International Foundation for Science (IFS) to build the wind tunnel.

\section{Author details}

'Departamento de Anatomia, Patologia e Clínicas Veterinárias, Escola de Medicina Veterinária e Zootecnia, Universidade Federal da Bahia, Av. Ademar de Barros 500, 40170-000 Salvador, BA, Brazil. ²Departamento de Química, Universidade Federal de São Carlos, 13565-905 São Carlos, SP, Brazil.

${ }^{3}$ Departamento de Ciências Biológicas, Faculdade de Ciências Farmacêuticas, Universidade Estadual Julio de Mesquita Filho, UNESP, 14801-902 Araraquara, SP, Brazil. ${ }^{4}$ Departamento de Estatística Matemática Aplicada E Computação Universidade Estadual Paulista Júlio de Mesquita Filho, Instituto de Geociências e Ciências Exatas de Rio Claro, v 24 A, 1515, Bela Vista, 13506-970 Rio Claro, SP, Brazil.

Received: 7 November 2013 Accepted: 31 January 2014

Published: 6 February 2014

\section{References}

1. Bray D, Bandi K, Brazil R, Oliveira A, Hamilton J: Synthetic sex pheromone attracts the leishmaniasis vector Lutzomyia longipalpis (Diptera: Psychodidae) to traps in the field. J Med Entomol 2009, 46:428-434.

2. Andrade AJ, Andrade MR, Dias ES, Pinto MC, Eiras AE: Are light traps baited with kairomones effective in the capture of Lutzomyia longipalpis and Lutzomyia intermedia? An evaluation of synthetic human odor as an attractant for phlebotomine sandflies (Diptera: Psychodidae: Phlebotominae). Mem Inst Oswaldo Cruz 2008, 103(4):337-343.

3. Hall D, Beevor P, Cork A, Nesbitt B, Vale G: A potent olfactory stimulant and attractant for tsetse isolated from cattle odours. Insect Sci App/ 1984, 5:335-339.

4. Bernier UR, Kline DL, Barnard DR, Schreck CE, Yost RA: Analysis of human skin emanations by gas chromatography/mass spectrometry: 2: identification of volatile compounds that are candidate attractants for the yellow fever mosquito (Aedes aegypti). Analyt Chem 2000, 72(4):747-756.

5. Essen PV, Kemme J, Ritchie S, Kay B: Differential responses of Aedes and Culex mosquitoes to octenol or light in combination with carbon dioxide in Queensland, Australia. Med Vet Entomol 1994, 8(1):63-67.

6. Mann RS, Kaufman PE, Butler JF: Lutzomyia spp. (Diptera: Psychodidae) response to olfactory attractant-and light emitting diode-modified Mosquito Magnet X (MM-X) traps. J Med Entomol 2009, 46(5):1052-1061.

7. Pinto M, Barbieri K, Silva M, Graminha M, Casanova C, Andrade A, Eiras A: Octenol as attractant to Nyssomyia neivai (Diptera: Psychodidae: Phlebotominae) in the field. J Med Entomo/ 2011, 48(1):39-44.

8. Sant'ana AL, Eiras AE, Cavalcante RR: Electroantennographic responses of the Lutzomyia (Lutzomyia) longipalpis (Lutz \& Neiva) (Diptera: Psychodidae) to 1-octen-3-ol. Neotrop Entomol 2002, 31:13-17.

9. Meijerink J, Braks M, Brack A, Adam W, Dekker T, Posthumus M, Van Beek T, Van Loon J: Identification of olfactory stimulants for Anopheles gambiae from human sweat samples. J Chem Ecol 2000, 26(6):1367-1382.

10. Mathew N, Ayyanar E, Shanmugavelu S, Muthuswamy K: Mosquito attractant blends to trap host seeking Aedes aegypti. Parasitol Res 2013, 112(3):1-8.

11. Galati E: Phylogenetic systematics of Phlebotominae (Diptera, Psychodidae) with emphasis on American groups. Bol Dir Malario/ San Amb 1995, 35(Supl 1):133-142. 
12. Young DG, Duran MA: Guide to the identification and geographic distribution of Lutzomyia sandflies in Mexico, the West Indies, Central and South America (Diptera: Psychodidae). In DTIC Document; 1994.

13. Pinto MC, Bray DP, Eiras AE, Carvalheira HP, Puertas CP: Attraction of the cutaneous leishmaniasis vector Nyssomyia neivai (Diptera: Psychodidae) to host odour components in a wind tunnel. Parasit Vectors 2012, 5(1):210

14. Cilek J, Ikediobi C, Hallmon C, Johnson R, Onyeozili E, Farah S, Mazu T, Latinwo L, Ayuk-Takem L, Bernier U: Semi-field evaluation of several novel alkenol analogs of 1-octen-3-ol as attractants to adult aedes albopictus and culex quinquefasciatus. J Am Mosa Control Assoc 2011, 27(3):256-262.

15. Xue R, Dong L, Zhang S, Deng C, Liu T, Wang J, Shen X: Investigation of volatile biomarkers in liver cancer blood using solid-phase microextraction and gas chromatography/mass spectrometry. Rapid Commun Mass Spectrom 2008, 22(8):1181-1186.

16. DeMilo A, Lee CJ, Levi V, Moreno D: Volatile components of a chicken feather hydrolystate that is highly attractive to the West Indian and Mexican fruit fly (Diptera: Tephritidae). J Entomol Sci 1997, 32:245-256.

17. Casanova C, Andrighetti MT, Sampaio SM, Marcoris ML, Colla-Jacques FE, Prado ÂP: Larval breeding sites of lutzomyia longipalpis (Diptera: Psychodidae) in visceral leishmaniasis endemic urban areas in Southeastern Brazil. PLoS Negl Trop Dis 2013, 7(9):e2443.

18. Sutcliffe JF, Shemanchuk JA, McKeown DB: Preliminary survey of odours that attract the black fly, Simulium arcticum (Malloch)(IIS-10.11)(Diptera: Simuliidae) to its cattle hosts in the Athabasca region of Alberta. Insect Sci App/ 1994, 15(4):487-494.

19. Vitta AC, Bohman B, Unelius CR, Lorenzo MG: Behavioral and electrophysiological responses of Triatoma brasiliensis males to volatiles produced in the metasternal glands of females. J Chem Ecol 2009, 35(10):1212-1221.

20. Birkett M, Agelopoulos N, Jensen K, Jespersen JB, Pickett J, Prijs $H$, Thomas G, Trapman J, Wadhams L, Woodcock C: The role of volatile semiochemicals in mediating host location and selection by nuisance and disease-transmitting cattle flies. Med Vet Entomol 2004, 18(4):313-322.

21. Jhumur US, Dötterl S, Jürgens A: Floral odors of Silene otites: their variability and attractiveness to mosquitoes. J Chem Ecol 2008, 34(1):14-25.

22. Jong S, Birmingham J: Mushrooms as a source of natural flavor and aroma compounds. In Mushroom Biology and Mushroom Products. Edited by Chang ST, Chang ST, Buswell JA, Chiu SW. ; 1993:345-366.

23. Yang $Y$, Nan $H$, Wang G, Yang W, X J: Comparative determination of the volatile components of prunella vulgaris $\mathrm{L}$ : from different geographical origins by headspace solid-phase microextraction and gas chromatography-mass spectrometry. Anal Lett 2013, 46(13):2001-2016

24. Schlein Y, Muller G: Assessment of plant tissue feeding by sandflies (Diptera: Psychodidae) and mosquitoes (Diptera: Culicidae). J Med Entomol 1995, 32(6):882-887.

25. Spiegel CN, Oliveira SM, Brazil RP, Soares MJ: Structure and distribution of sensilla on maxillary palps and labella of Lutzomyia longipalpis (Diptera: Psychodidae) sandflies. Microsc Res Tech 2005, 66(6):321-330.

doi:10.1186/1756-3305-7-60

Cite this article as: Magalhães-Junior et al: A laboratory evaluation of alcohols as attractants for the sandfly Lutzomyia longipalpis

(Diptera:Psychodidae). Parasites \& Vectors 2014 7:60.

\section{Submit your next manuscript to BioMed Central and take full advantage of:}

- Convenient online submission

- Thorough peer review

- No space constraints or color figure charges

- Immediate publication on acceptance

- Inclusion in PubMed, CAS, Scopus and Google Scholar

- Research which is freely available for redistribution 